\title{
Composição química e avaliação da atividade antibacteriana e toxicidade do óleo essencial de Croton zehntneri (variedade estragol)
}

\author{
José G. M. da Costa, * Fabíola F. G. Rodrigues, Elissandra C. Angélico, Carla K. B. Pereira, \\ Erlânio O. de Souza, Germana F. R. Caldas, Monalisa R. Silva, Nara K. A. Santos, \\ Magaly L. Mota, Paula F. dos Santos
}

Departamento de Química Biológica, Laboratório de Pesquisa de Produtos Naturais, Universidade Regional do Cariri, Rua Cel. Antônio Luiz 1161, Pimenta, 63105-000 Crato-CE, Brasil

\begin{abstract}
RESUMO: Tendo em vista que bactérias resistentes a antimicrobianos representam um desafio no tratamento de infecções, é notória a necessidade de encontrar novas substâncias com propriedades antimicrobianas para serem utilizadas no combate a esses microrganismos. Este trabalho relata a avaliação da atividade antibacteriana, toxicidade e identificação dos componentes químicos do óleo essencial de Croton zehntneri (variedade estragol), planta utilizada na medicina popular como calmante e estimulante do apetite. A atividade antimicrobiana e a concentração inibitória mínima (CIM) foram determinadas pelo método de difusão em discos. A avaliação da toxicidade foi realizada frente à Artemia salina com resultado considerado ativo $\left(\mathrm{CL}_{50}<100 \mu \mathrm{g} / \mathrm{mL}\right)$. O óleo essencial das folhas apresentou atividade antibacteriana frente a todas as bactérias testadas exceto contra Salmonella typhimurium, sendo o melhor resultado frente a Shigella flexneri com CIM de $50 \mu \mathrm{g} / \mathrm{mL}$. A análise da composição química foi obtida por cromatografia gasosa acoplada a espectrometria de massa (CG/EM) e permitiu identificar um total de $97,4 \%$ dos componentes, com presença majoritária de estragol $(76,8 \%)$. A presença de tal constituinte nos impulsiona a realização de estudos com outras bactérias, já que o estragol foi anteriormente relatado como sendo responsável por atividades antibacterianas.
\end{abstract}

Unitermos: Croton zehntneri, Euphorbiaceae, estragol, óleo essencial, atividade antibacteriana, toxicidade, CIM.

\begin{abstract}
Chemical composition and evaluation of the antibacterial activity and toxicity of the essential oil of Croton zehntneri (variety estragol)". Observing that bacteria resistant to antimicrobials represent a challenge in the treatment of infections, it is notorious the need of finding new substances with antimicrobial features to be used in the fight against these microorganisms. This work relates the evaluation of the antibacterial activity, toxicity and identification of the chemical components of the essential oil of Croton zehntneri (variety estragol), plant used in the popular medicine as tranquilizer and appetite stimulant. The antimicrobial activity and minimum inhibitory concentration (MIC) were determined by the method of diffusion in discs. The evaluation of the toxicity was held through brine shrimp test with results considered active $\left(\mathrm{LC}_{50}<100 \mu \mathrm{g} / \mathrm{mL}\right)$. The essential oil of leaves presented antibacterial activity with all the bacteria tested except with Salmonlla typhimurium, being the best result with the Shigella flexneri with MIC of $50 \mu \mathrm{g} / \mathrm{mL}$. The analysis of the chemical composition was obtained by gas chromatography coupled to mass spectrometry (GC/MS) and permitted to identify a total of 97.4 $\%$ of the components, with major presence of estragol $(76.8 \%)$. The presence of the latter drives us to studies with other bacteria, as the estragol was previously reported as being responsible for antibacterial activities.
\end{abstract}

Keywords: Croton zehntneri, Euphorbiaceae, estragol, essential oil, antibacterial activity, toxicity, MIC.

\section{INTRODUÇÃO}

O desenvolvimento de resistência aos antimicrobianos por diversos patógenos vem aumentando a cada dia, tornando-se de grande importância a pesquisa de novos agentes para o combate de infecções. Novas fontes de substâncias obtidas de espécies nativas do Brasil, que apresentem atividade antimicrobiana e baixa toxicidade, podem vir a ser uma alternativa viável, de baixo custo e de fácil acesso pelas comunidades carentes, da região onde tais espécies são encontradas. As plantas produzem substâncias para sua própria 
defesa em resposta a um ataque patogênico, muitas sintetizam e emitem inúmeros compostos voláteis com a finalidade de defesa e atrair seus polinizadores (Costa et al., 2005).

A família Euphorbiaceae compreende cerca de 300 gêneros e 7600 espécies (Farnsworth et al., 1969; Cronquist, 1981), possuindo hábito heterogêneo, onde se incluem árvores, arbustos, ervas e trepadeiras (Leme, 1994). O gênero Croton destaca-se por seu expressivo número de espécies (750 a 800), de distribuição neotropical (Judd et al., 1999; Heluani et al., 2000), com poucos representantes paleotropicais (Webster, 1994) tendo importância econômica. Várias espécies de Croton apresentam óleos essenciais e constituintes ativos como terpenóides, flavonóides e alcalóides, sendo com freqüência utilizada na medicina popular. Algumas espécies possuem propriedades terapêuticas já comprovadas (Santos et al., 2005; Palmeira Júnior et al., 2006; Souza et al., 2006; Perazzo et al., 2007; Torrico et al., 2007; Rocha et al., 2008).

Croton zehntneri Pax et Hoffm., conhecida popularmente como "canela de cunhã", "canelinha" ou "canela-brava", é uma planta subarbustiva e caducifólia do Nordeste brasileiro, cujas folhas e talos são dotadas de um aroma que lembra uma mistura de erva-doce e cravo-da-Índia. Entretanto, este aroma mostra-se variável entre exemplares desta planta coletados em diferentes localidades do Nordeste. Isto se deve à variação na concentração dos constituintes químicos mais abundantes nos seus óleos essenciais (Craveiro et al., 1978). Assim, distinguem-se para esta espécie quatro tipos químicos como: anetol - para os exemplares coletados em Fortaleza (CE) e Viçosa (CE); eugenol - para os coletados em Areia Branca (RN) e Quixadá (CE); metil-eugenol - para os coletados em Ipu (CE) e Oeiras (PI); estragol - para os exemplares coletados em Tianguá (CE) e Granja (CE) (Morais et al., 2006).

C. zehntneri é usado na medicina popular principalmente como sedativo, como estimulante de apetite e para aliviar distúrbios intestinais (Matos, 2000; Agra et al., $2007 \&$ 2008), sendo comprovados os efeitos antioxidante (Morais et al., 2006), atividade antinoceptiva (Oliveira et al., 2001) e efeitos depressivos sobre o sistema nervoso central em ratos e camundongos (Batatinha et al., 1995) do óleo essencial de suas folhas.

Considerando-se que não há estudos sobre o potencial microbiológico de $C$. zehntneri (variedade estragol) e que estudos sobre a atividade antimicrobiana de plantas medicinais apresentam um grande desafio para a descoberta e identificação de novas substâncias ativas, este trabalho teve por objetivo avaliar preliminarmente o potencial da atividade antibacteriana e a toxicidade do óleo essencial dessa espécie.

\section{MATERIAL E MÉTODOS}

584 Rev. Bras. Farmacogn.

Braz J. Pharmacogn.

18(4): Out./Dez. 2008

\section{Material vegetal e obtenção do óleo essencial}

Folhas de C. zehntneri foram coletadas no mês de março/2006 no Morro do Chapéu, em Salitre (Ce). Uma amostra representativa foi classificada e depositada no Herbário Caririense Dárdaro de Andrade Lima da Universidade Regional do Cariri, sob o número 1619.

O óleo essencial das folhas secas $(425 \mathrm{~g})$ foi obtido por hidrodestilação, utilizando aparelho tipo Clevenger, por um período de duas horas. Em seguida seco com sulfato de sódio anidro obtendo rendimento de $1,56 \%$ e mantido em refrigerador até a análise.

\section{Análise da composição química}

Aidentificação dos componentes majoritários do óleo essencial das folhas de C. zehntneri foi obtida através de cromatografia gasosa acoplada a espectrometria de massa (CG/EM), em um espectrômetro Hewlett-Packard modelo 5971, operando com energia de ionização de 70 eV. Utilizou-se coluna capilar de sílica fundida DB-5 (30 m x 0,25 mm d.i., 0,25 $\mu \mathrm{m}$ de espessura do filme) e carreador de gás hélio com fluxo de $1 \mathrm{~mL} / \mathrm{min}$ com split. As temperaturas do injetor e detector foram programadas de $250{ }^{\circ} \mathrm{C}$ e $200{ }^{\circ} \mathrm{C}$, respectivamente. A temperatura da coluna foi determinada de $35^{\circ} \mathrm{C}$ a $180^{\circ} \mathrm{C}$ a $4^{\circ} \mathrm{C} / \mathrm{min}$ e, em seguida de $180^{\circ} \mathrm{C}$ a $280^{\circ} \mathrm{C}$ a $10^{\circ} \mathrm{C} / \mathrm{min}$. Os espectros de massa foram obtidos de 30 a $450 \mathrm{~m} / \mathrm{z}$. Componentes individuais foram identificados por correspondências de seus espectros de massa com os da base de dados, bem como através de comparação visual da fragmentação padrão com aqueles relatados na literatura (Adams, 2001).

\section{Avaliação da toxicidade}

A toxicidade foi avaliada frente a Artemia salina Leach, através do método proposto por Meyer et al. (1982). O teste foi feito em triplicata com diferentes concentrações $(250,125,100,75 \mu \mathrm{g} / \mathrm{mL})$, acompanhado de um controle positivo preparado com água marinha e dicromato de potássio $\left(\mathrm{K}_{2} \mathrm{Cr}_{2} \mathrm{O}_{7}\right)$, e de um controle negativo com água marinha e Tween 80 . A contagem do número de larvas mortas foi realizada após 24 horas e esse número foi usado para o calculo da $\mathrm{CL}_{50}$ pelo método de regressão linear, sendo considerado ativo quando apresentam valores de $\mathrm{CL}_{50}<1000 \mu \mathrm{g} / \mathrm{mL}$ (Meyer et al, 1982).

\section{Avaliação da atividade antibacteriana e concentração inibitória mínima (CIM)}

$\mathrm{Na}$ realização da atividade antibacteriana foram utilizadas cinco culturas padrões cedidas pela Fundação Oswaldo Cruz - FIOCRUZ, sendo três gramnegativas Shigella flexneri (ATCC 12022), Salmonella typhimurium (BM/PANAMA - TY2) e Escherichia coli 
(ATCC 25922) e duas gram-positivas Sthaphylococcus aureus (ATCC 10390) e Streptococus $\beta$-haemolyticus (ATCC 6314), reavivadas em meio Brain Heart Infusion (BHI ) e incubadas por 24 horas a $37^{\circ} \mathrm{C}$.

A ação antimicrobiana e a concentração inibitória mínima foram determinadas pelo método de difusão em Agar (Bauer et al., 1966) adaptado por Romeiro (2001) e Koneman et al. (1993). As bactérias foram replicadas, com auxílio de um swab estéril, em placas de petri previamente preparadas com ágar Muller-Hilton onde discos de papel estéreis $(6 \mathrm{~mm}$ de diâmetro) foram impregnados com $20 \mu \mathrm{L}$ da solução do óleo, em concentrações de 1000, 500, 100, 50 e 25 $\mu \mathrm{g} / \mathrm{mL}$. O ensaio foi realizado em triplicata utilizando como controles positivos ampicilina $(100 \mu \mathrm{g} / \mathrm{mL})$ e cloranfenicol $(100 \mu \mathrm{g} / \mathrm{mL})$ e como controle negativo Tween 80 e água destilada. As placas foram encubadas em estufa a $37^{\circ} \mathrm{C}$ e os resultados foram lidos após 24 horas. O CIM foi considerado a menor concentração do óleo que inibiu completamente o crescimento bacteriano.

\section{RESULTADOS E DISCUSSÃO}

O cromatograma do óleo essencial de $C$. zehntneri mostrou que doze picos foram detectados. Foram identificados $97,4 \%$ dos constituintes químicos (Tabela 1) dentre mono- e sesquiterpenos, sendo reconhecido como componente majoritário o estragol, representando $76,8 \%$ do teor do óleo essencial.

Os índices de mortalidade das larvas no bioensaio de toxicidade do óleo essencial sobre Artemia salina variaram entre 0 e $100 \%$. A dose necessária para matar $50 \%$ das larvas $\left(\mathrm{CL}_{50}\right)$ foi calculada em $100 \mu \mathrm{g} / \mathrm{mL}$, indicando que esse óleo possui atividade considerada significativa por apresentar $\mathrm{CL}_{50}$ menor que $1000 \mu \mathrm{g} / \mathrm{mL}$ (Meyer et al., 1982).

No teste de susceptibilidade para a avaliação

Tabela 1. Composição química percentual do óleo essencial das folhas de C. zehntneri.

\begin{tabular}{lcc}
\hline Constituintes & Tempo de Retenção (min) & Porcentagem (\%) \\
\hline Estragol & 28,8 & 76,8 \\
1,8-Cineol & 21,8 & 7,0 \\
Eugenol & 35,0 & 5,3 \\
Mirceno & 19,6 & 4,4 \\
Biciclogermacreno & 37,7 & 1,7 \\
Beta-Ocimeno & 22,2 & 1,6 \\
Sabineno & 19,2 & 0,61 \\
\hline
\end{tabular}

Tabela 2. Halos de inibição obtidos para o óleo essencial de das folhas de C. zehntneri contra bactérias patogênicas.

\begin{tabular}{lcccc}
\hline & \multicolumn{4}{c}{ Halo de inibição (mm de diâmetro) } \\
\cline { 2 - 5 } Microrganismos & $\begin{array}{c}\text { Óleo essencial }(1000 \\
\mu \mathrm{g} / \mathrm{mL})\end{array}$ & $\begin{array}{c}\text { Ampicilina } \\
(100 \mu \mathrm{g} / \mathrm{mL})\end{array}$ & $\begin{array}{c}\text { Cloranfenicol }(100 \\
\mu \mathrm{g} / \mathrm{mL})\end{array}$ & Água e Tween 80 \\
\hline Shigella flexneri & $15,33 \pm 0,47^{\mathrm{a}}$ & $20,66 \pm 0,94$ & $18,66 \pm 0,47$ & $\mathrm{ND}$ \\
Salmonella typhimurium & $\mathrm{ND}$ & $19,33 \pm 0,94$ & $20,66 \pm 0,94$ & $\mathrm{ND}$ \\
Sthaphylococcus aureus & $6,33 \pm 0,47^{\mathrm{b}}$ & $15 \pm 0,81$ & $25,33 \pm 0,47$ & $\mathrm{ND}$ \\
Escherichia coli & $6,66 \pm 0,47^{\mathrm{b}}$ & $25,33 \pm 0,47$ & $22,66 \pm 0,47$ & $\mathrm{ND}$ \\
Streptococus $\beta$-haemolyticus & $6,66 \pm 0,47^{\mathrm{b}}$ & $17,66 \pm 0,47$ & $19 \pm 0,47$ & $\mathrm{ND}$ \\
\hline
\end{tabular}

(*) A inibição ocorreu somente no ponto de aplicação; $\mathrm{ND}=$ Não detectável. Médias seguidas de letras iguais, na coluna, não diferem significativamente entre si, pelo teste de Tukey a $5 \%$ de probabilidade.

Tabela 3. Concentração Inibitória Mínima (CIM) do óleo essencial das folhas de C. zehntneri.

\begin{tabular}{lccccc}
\hline \multirow{2}{*}{ Microrganismos } & \multicolumn{5}{c}{ Concentração do óleo $(\mu \mathrm{g} / \mathrm{mL})$} \\
\cline { 2 - 5 } & 1000 & 500 & 100 & 50 & 25 \\
\hline S. flexneri & $15,33 \pm 0,47$ & $10 \pm 0,00$ & $8,33 \pm 0,47$ & $8,00 \pm 0,81$ & $6,33 \pm 0,47$ \\
S. typhimurium & $\mathrm{ND}$ & $\mathrm{ND}$ & $\mathrm{ND}$ & $\mathrm{ND}$ & $\mathrm{ND}$ \\
S. aureus & $6,33 \pm 0,47$ & $6 \pm 0,81$ & $\mathrm{ND}$ & $\mathrm{ND}$ & $\mathrm{ND}$ \\
E. coli & $6,66 \pm 0,47$ & $6 \pm 0,81$ & $\mathrm{ND}$ & $\mathrm{ND}$ & $\mathrm{ND}$ \\
S. $\beta$-haemolyticus & $6,66 \pm 0,47$ & $6 \pm 0,81$ & $\mathrm{ND}$ & $\mathrm{ND}$ & ND \\
\hline & & & & Rev. Bras. Farmacogn. & Braz J. Pharmacogn. \\
& & & & 585 \\
& & & & 18(4): Out./Dez. 2008
\end{tabular}


da atividade antibacteriana, o óleo essencial das folhas apresentou atividade frente a todas as bactérias testadas exceto contra Salmonella typhimurium, sendo o melhor resultado frente à Shigella flexneri com maior halo de inibição de $15 \mathrm{~mm}$. Com as linhagens Sthaphylococcus aureus, Escherichia coli e Streptococus $\beta$-haemolyticus foram observados discretos halos de $6 \mathrm{~mm}$ (Tabela 2). Pela análise estatística (ANOVA), foi verificado que quando comparados os halos de inibição obtidos para Sthaphylococcus aureus, Escherichia coli e Streptococus $\beta$-haemolyticus, não houve diferença significativa.

Em relação à avaliação da concentração inibitória mínima, o óleo essencial apresentou atividade significativa apenas contra a linhagem de Shigella flexneri demonstrando sensibilidade nas concentrações de $1000,500,100$ e $50 \mu \mathrm{g} / \mathrm{mL}$, com CIM de $50 \mu \mathrm{g} / \mathrm{mL}$ (Tabela 3).

O óleo essencial das folhas de Croton zehntneri é constituido principalmente de monoterpenóides e sesquiterpenóides, dentre seus constituintes, demonstrouse a presença majoritária de estragol $(76,8 \%)$.

Diante dos resultados dos bioensaios preliminares o óleo essencial demonstrou potencial biológico contra bactérias patogênicas e toxicidade ativa, incentivando assim novas pesquisas com substâncias isoladas dessa espécie, na busca de alternativas terapêuticas que possam servir de subsídio para novas fontes racionais a partir de produtos naturais brasileiros.

\section{AGRADECIMENTOS}

A FUNCAP e ao CNPq pelo apoio financeiro e a Fundação Oswaldo Cruz (FIOCRUZ), pela concessão das bactérias padrões.

\section{REFERÊNCIAS}

Adams RP 2001. Identification of essencial oil components by gas chromatography/massa spectroscopy. Allured.

Agra MF, França PF, Barbosa-Filho JM 2007. Synopsis of the plants known as medicinal and poisonous in Northeast of Brazil. Rev Bras Farmacogn 17: 114140.

Agra MF, Silva KN, Basílio IJLD, França PF, Barbosa-Filho JM 2008. Survey of medicinal plants used in the region Northeast of Brazil. Rev Bras Farmacogn 18: 472-508.

Batatinha MJM, Souza-Spinosa H, Bernardi MM 1995. Croton zehntneri: possible central nervous system effects of the essencial oil in rodents. $J$ Ethnopharmacol 45: 53-57.

Bauer AW, Kirby WMM, Sherris JC, Turck M 1966. Antibiotic susceptibility festing by a standartized single disc melhod. Am J Clin Patol 45: 493-496.

Costa JGM, Rodrigues FFG, Angélico EC, Silva MR, Mota ML, Santos NKA, Cardoso ALH, Lemos TLG 2005. Estudo químico-biológio dos óleos essenciais de Hyptis martiusii, Lippia sidoides e Syzigium aromaticum frente a larvas do Aedes aegypti. Rev Bras Farmacogn 15: 304-309.

Craveiro AA, Andrade CHS, Matos FJA, Alencar JW 978. $J$
Agric Food Chem 26: 773.

Cronquist A 1981. An integrated system of classification of flowering plants. New York: Columbia Universty Press., p. 1262

Farnsworth NR, Blomster RN, Mesmmer WM 1969. A phytochemical and biological review of the genus Croton. Lloydia 32: 1-28.

Heluani CS, Catalan CAN, Hernández LR, Tapia EB, Natan PT, 2000. Three new diterpenoids bazed on novel sarcopetalene skeleton from Croton sarcopetalus. $J$ Nat Prod 63: 222-225.

Judd WS, Campbell CS, Kellog EA, Stevens PF 1999. Plant Systematics: a Phylogenetic Approach. Massachusetts: Sinauer Associates.

Koneman EW, Allen SD, Dowwel-Junior VR, Sommers HM 1993. Diagnóstico Microbiológico - Texto e Atlas Colorido. 2 ed. São Paulo: Medicina Panamericana Editora do Brasil Ltda.

Leme CLD 1994. Anatomia Comparada do lenho do caule, raiz e ramo de algumas espécies de Euphorbiaceae da Mata Atlântica, Dissertação de Mestrado, Instituto de Biociências-USP, São Paulo. 73 p.

Matos FJA 2000. Plantas Medicinais. 2 ed. Fortaleza:Editora UFC.

Meyer BN, Ferrigne NR, Putnam JE, Jacobsen LB, Nichols DE, Mclaughlin JE 1982. Brine shrimp: A convenient general bioassay for active plant constituents. Planta Med 45: 31-34.

Morais SM, Catunda Júnior FEA, Silva ARA, Stone J, Martins Neto RD, Cardoso JHL 2006. Atividade antioxidante de óleos essenciais de espécies de croton do Nordeste do Brasil. Quim Nova 29: 907-910.

Oliveira AC, Leal-Cardoso JH, Santos CF, Morais SM, Coelho-de-Sousa AN 2001. Antinociceptive effects of the essential oil of Croton zehntneri in mice. Braz J Med Biol Res 34: 1471-1474.

Palmeira Júnior SF, Alves VL, Moura FS, Vieira LFA, Conserva LM, Lemos RPL Constituintes químicos das folhas e caule de Croton sellowii (Euphorbiaceae). Rev Bras Farmacogn 16: 397-402.

Perazzo FF, Carvalho JCT, Rodrigues M, Morais EKL, Macie MAM 2007. Comparative anti-infammatory and antinociceptive effects of terpenoids and an aqueous extract obtained from Croton cajucara Benth. Rev Bras Farmacogn 17: 521-528.

Rocha FF, Neves EMN, Costa EA, Matos LG, Müller AH, Guilhon GMSP, Cortes WS, Vanderlinde FA 2008. Evaluation of antinociceptive and antiinflammatory effects of Croton pullei var. glabrior Lanj. (Euphorbiaceae). Rev Bras Farmacogn 18: 344-349.

Romeiro RS 2001. Métodos em Bacteriologia de Plantas. Viçosa: Editora UFC.

Santos PML, Schripsema J, Kuster RM 2005. Flavonóides $O$-glicosilados de Croton campestris St. Hill. (Euphorbiaceae). Rev Bras Farmacogn 15: 321-325.

Souza MAA, Souza SR, Veiga Jr VF, Cortez JKPC, Leal RS, Dantas TNC, Maciel MAM 2006. Composição química do óleo fixo de Croton cajucara e determinação das suas propriedades fungicidas. Rev Bras Farmacogn 16 (Supl.): 599-610.

Torrico F, Cepeda M, Guerrero G, Melendez F, Blanco Z, Canelón DJ, Diaz B, Compagnone RS, Suárez AI 2007. Hypoglycaemic effect of Croton cuneatus in streptozotocin-induced diabetic rats. Rev Bras Farmacogn 17: 166-169.

Webster GL 1994. Systematics of the Euphorbiaceae. Annals of the Missouri Botanical Garden 1: 144. 